\title{
Free Energy on a Cycle Graph and Trigonometric Deformation of Heat Kernel Traces on Odd Spheres
}

\author{
Nahomi Kan \\ National Institute of Technology, Gifu College, Motosu-shi, Gifu 501-0495, Japan \\ and \\ Kiyoshi Shiraishi \\ Graduate School of Sciences and Technology for Innovation, \\ Yamaguchi University, Yamaguchi-shi, Yamaguchi 753-8512, Japan
}

July 19, 2018

\begin{abstract}
We consider a possible 'deformation' of the trace of the heat kernel on odd dimensional spheres, motivated by the calculation of the free energy of a scalar field on a discretized circle. By using an expansion in terms of the modified Bessel functions, we obtain the values of the free energies after a suitable regularization.

PACS: 02.10.Ox, 02.20.Uw, 04.60.Nc, 04.62.+v, 11.10.Kk.
\end{abstract}

\section{Introduction}

Evaluation of functional determinants is an important task in quantum field theories [1]. To obtain the value of an infinite dimensional determinant, we need appropriate regularization.

The heat kernel is one of the most powerful tools in mathematical physics and spectral geometry $[2,3,4,5,6,7]$. We can regard the trace of the heat kernel (the heat kernel trace) as a mathematical basis. Its advantage is that it can be computed at once from the spectrum of the Laplacian operator defined in the theory. The trace of the heat kernel is given by the sum of the Laplacian eigenvalues exponentiated with the Schwinger "proper time' $t$.

The equivalence in computing the determinant by using the heat kernel trace and by the zeta function technique $[8,9]$ can be illustrated as follows. The spectral zeta function $\zeta_{S}(s)$ for a differential operator on a compact space with discrete eigenvalues $\lambda_{l}$ and degeneracies (multiplicities) $g_{l}$ is defined by the summation

$$
\zeta_{S}(s) \equiv \sum_{l} g_{l} \lambda_{l}^{-s}
$$

and the log of the determinant is formally obtained by

$$
-\zeta_{S}^{\prime}(0)=-\left.\frac{\partial}{\partial s} \zeta_{S}(s)\right|_{s=0}=\ln \prod_{l} \lambda_{l}^{g_{l}}
$$


The spectral zeta function is written in an integral representation

$$
\zeta_{S}(s)=\frac{\mu_{R}^{2 s}}{\Gamma(s)} \int_{0}^{\infty} \frac{d t}{t^{1-s}} \sum_{l} g_{l} \exp \left(-\lambda_{l} t\right),
$$

where $\mu_{R}$ is a renormalization parameter and $\Gamma(z)$ is the gamma function. In this expression,

$$
\kappa(t)=\sum_{l} g_{l} \exp \left(-\lambda_{l} t\right)
$$

is the trace of the heat kernel.

The one-loop free energy is then obtained by computing the log of the determinant for the operator. Once the spectral zeta function $\zeta_{S}(s)$ is known, the contribution of the field under consideration to the free energy can be obtained.

It is known that the renormalization parameter $\mu_{R}$ only appear in even dimensional space. Namely, $\zeta_{S}(0)$ vanishes identically in odd dimensional space [10]. Because $\Gamma(s) \sim \frac{1}{s}$ for small $s$, the one-loop free energy of a scalar field $\phi$ on an odd dimensional space can be evaluated as

$$
F=-\ln Z=-\ln (\operatorname{Det} D)^{-1 / 2}=-\frac{1}{2} \int_{0}^{\infty} \frac{d t}{t} \operatorname{Tr} \exp (-D t) .
$$

Here $Z$ is the partition function defined through the path integral

$$
Z=\int \mathcal{D} \phi e^{-S}
$$

where $S$ is the quadratic action for the scalar field described by

$$
S=\frac{1}{2} \int d^{d} x \phi(x) D \phi(x) .
$$

Equation (5) can also be understood using an 'identity'1

$$
\ln \lambda^{\prime}=\int_{0}^{\infty} \frac{d t}{t} e^{-\lambda t}
$$

for each positive eigenvalue $\lambda$ of the operator $D$. This identity is correct up to an additional infinite constant, which does not depend on $\lambda$ and thus is irrelevant for physical quantities. The infinity should be regularized by appropriate methods.

In this paper, we first review the derivation of the free energy of a scalar field on a circle $S^{1}$ by using the heat kernel trace. The Laplacian on $S^{1}$ has infinite discrete eigenvalues.

The discretization of space is discussed from time to time, in various context, such as approaches to quantum gravity $[11,12,13,14]$, lattice (or coarse-graining) simulations of field theory [15], some exact supersymmetric models [16, 17, 18], etc. A naive discretization of $S^{1}$ corresponds to defining a field theory on a cycle graph $C_{N}$ [19], where $N$ is the number of vertices. The eigenspectrum of the graph Laplacian of $C_{N}$ is known to be finite and discrete. In the present paper, we show the regularization procedure of the free energy on $C_{N}$ by using an expansion of the heat kernel trace in terms of the modified Bessel functions. A key point of this expansion is the fact that the eigenvalues of the graph Laplacian on $C_{N}$ can be written by the trigonometric functions, such as $\propto 4 \sin ^{2} \frac{\pi p}{N}$ $(p=0,1, \ldots N-1)$.

Motivated by this calculation, we come to the idea to deform the eigenvalues as well as degeneracies in heat kernel traces on higher dimensional space, by use of trigonometric

\footnotetext{
${ }^{1}$ This can be confirmed by differentiating both sides of the equation by $\lambda$.
} 
functions. We demonstrate the trigonometric deformation of the heat kernel traces and obtain regularized free energies on odd dimensional spheres in the present paper.

Our trigonometric deformation is almost equivalent to $q$-deformation [20]. ${ }^{2}$ The (symmetric) $q$-number is defined by ${ }^{3}$

$$
[p]=\frac{q^{p / 2}-q^{-p / 2}}{q^{1 / 2}-q^{-1 / 2}} .
$$

In the 'classical' limit $q \rightarrow 1,[p]=p$ is recovered. If $q$ is chosen as a primitive root of unity of degree $N$, i.e., $q=e^{i \frac{2 \pi}{N}}$, it is found out to be

$$
[p]=\frac{\sin \frac{\pi p}{N}}{\sin \frac{\pi}{N}} .
$$

There is, however, an ambiguity of the interpretation in extensions. We give a comment on this ambiguity later in the present paper.

The outline of this paper is as follows. In Section 2 we calculate the free energy of a scalar field on $S^{1}$ by utilizing the heat trace method. Section 3, we consider the scalar field on $C_{N}$ and develop the regularization technique of the free energy. In Section 4 we propose a trigonometric deformation of the heat kernel traces of scalar fields on odd spheres. In Section 5 we perform an explicit calculation of the free energy of a scalar field on $S^{3}$ and calculations of the free energies by the deformed heat kernel traces with a suitable regularization. A comment on the ambiguity in extensions is given in Section 6 . Finally, Section 7 offers some conclusions and directions for future research.

\section{$2 \quad$ Free energy on $S^{1}$}

We begin by the following Euclidean action for a massive scalar field with mass $m$ on $S^{1}$ :

$$
S=\frac{1}{2} \int d x\left(\phi^{\prime 2}+m^{2} \phi^{2}\right)
$$

where we denote the derivative with respect to $x$ by the prime $\left(^{\prime}\right)$. If the periodic condition $\phi(x)=\phi(x+2 \pi a)$ is assumed, i.e., $a$ is the radius of the circle, the eigenvalue of the operator $D=-\partial_{x}^{2}+m^{2}$ is found to be

$$
\lambda_{l}(m)=\frac{l^{2}}{a^{2}}+m^{2}
$$

where $l$ is an integer $(l \in \mathbb{Z})$.

Now, we compute the free energy by the heat kernel method. To this purpose, recall the relation of one-loop partition function with traced heat kernel and we get the expression

$$
F(m)=-\ln Z=-\frac{1}{2} \int_{0}^{\infty} \frac{d t}{t} \sum_{l \in \mathbb{Z}} e^{-a^{2} \lambda_{l}(m) t} .
$$

Here, we note that we take $a^{2} \lambda_{l}(m)$ in stead of $\lambda_{l}(m)$ in the exponential function because of a reparametrization invariance on $t$. Then, the calculation is straightforward as

$$
F(m)=-\frac{1}{2} \int_{0}^{\infty} \frac{d t}{t} \sum_{l \in \mathbb{Z}} e^{-\left(l^{2}+m^{2} a^{2}\right) t}
$$

\footnotetext{
${ }^{2}$ As a mathematical term, the word 'deformation' usually represents for varying algebraic relations. In this paper, 'deformation' is used rather in a general sense.

${ }^{3}$ Although the $q$-number is often denoted by $[p]_{q}$, the simpler notation $[p]$ is adopted throughout the present paper.
} 


$$
\begin{aligned}
& =-\frac{\sqrt{\pi}}{2} \int_{0}^{\infty} \frac{d t}{t^{3 / 2}} \sum_{l \in \mathbb{Z}} \exp \left(-m^{2} a^{2} t-\frac{\pi^{2} l^{2}}{t}\right) \\
& =-2 \sum_{l=1}^{\infty} \sqrt{\frac{m a}{l}} K_{1 / 2}(2 \pi m a l)-\frac{\sqrt{\pi}}{2} \int_{0}^{\infty} \frac{d t}{t^{3 / 2}} e^{-m^{2} a^{2} t} \\
& =-2 \sum_{l=1}^{\infty} \sqrt{\frac{m a}{l}} \sqrt{\frac{\pi}{4 \pi m a l}} e^{-2 \pi m a l}-\frac{\sqrt{\pi}}{2} \Gamma\left(-\frac{1}{2}\right) m a \\
& =-\sum_{l=1}^{\infty} \frac{e^{-2 \pi m a l}}{l}+\pi m a=\ln \left(1-e^{-2 \pi m a}\right)+\ln e^{\pi m a} \\
& =\ln (2 \sinh \pi m a) .
\end{aligned}
$$

Here we have used the Poisson summation formula

$$
\sum_{l \in \mathbb{Z}} e^{-l^{2} t}=\sqrt{\frac{\pi}{t}} \sum_{l \in \mathbb{Z}} e^{-\frac{\pi^{2} l^{2}}{t}},
$$

and the integration formula

$$
\begin{gathered}
K_{\nu}(z)=\frac{1}{2}\left(\frac{z}{2}\right)^{\nu} \int_{0}^{\infty} \exp \left(-t-\frac{z^{2}}{4 t}\right) t^{-\nu-1} d t, \\
K_{1 / 2}(z)=\sqrt{\frac{\pi}{2 z}} e^{-z},
\end{gathered}
$$

where $K_{\nu}(z)$ is the modified Bessel function of the second kind (Macdonald function) [21].

Incidentally, the regularized free energy on $S^{1}$ can also be directly obtained but if we introduce the mass scale $M$ of Pauli-Villars regularization [22], and is found to be

$$
F^{P V}=\frac{1}{2} \ln \prod_{l \in \mathbb{Z}} \frac{\lambda_{l}(m)}{\lambda_{l}(M)}=\ln \left(\frac{m}{M} \prod_{l=1}^{\infty} \frac{l^{2}+m^{2} a^{2}}{l^{2}+M^{2} a^{2}}\right)=\ln \frac{2 \sinh \pi m a}{2 \sinh \pi M a},
$$

and this reads

$$
F^{P V}=F(m)-F(M),
$$

and thus, we have confirmed the previous result of the heat kernel method.

In the next section, we consider the discretization of the circle and calculate the free energy on it. We will see the continuum limit recovers the free energy on $S^{1}$.

\section{$3 \quad$ Free energy on a cycle graph}

The cycle graph $C_{N}$ consists of $N$ vertices $(v \in V)$ and $N$ edges $(e \in E)$ connecting two adjacent vertices (see FIG. 1) [19].

We consider $N$ scalar degrees of freedom associated with $N$ vertices and define the action as follows:

$$
S=\frac{1}{2}\left(\frac{1}{a_{0}^{2}} \sum_{v, v^{\prime} \in V} \phi_{v} \Delta_{v v^{\prime}}^{G} \phi_{v^{\prime}}+\mu^{2} \sum_{v \in V} \phi_{v}^{2}\right),
$$

where $\Delta^{G}$ is the graph Laplacian for $C_{N}[23,24,25,26]$. This is an $N \times N$ matrix

$$
\Delta^{G}=\left(\begin{array}{rrrrrr}
2 & -1 & 0 & \cdots & 0 & -1 \\
-1 & 2 & -1 & \cdots & 0 & 0 \\
0 & -1 & 2 & \cdots & 0 & 0 \\
\vdots & \vdots & \vdots & \ddots & \vdots & \vdots \\
0 & 0 & 0 & \cdots & 2 & -1 \\
-1 & 0 & 0 & \cdots & -1 & 2
\end{array}\right) .
$$




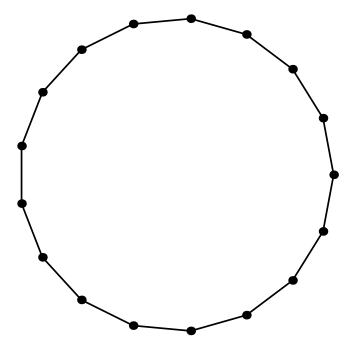

Figure 1: $C_{17}$ as an example of a cycle graph.

For later convenience, we define the 'mass' $\mu$ as

$$
\mu \equiv \frac{2}{a_{0}} \sinh \frac{m a_{0}}{2}
$$

Note that the action (20) becomes equivalent to the action (11) in the limit of $N \rightarrow \infty$ and $a_{0} \rightarrow 0$ while $a=N a_{0} /(2 \pi)$ is left constant.

Then, the eigenvalue of the operator $\Delta^{G}+\mu^{2}$ is expressed by

$$
\bar{\lambda}_{p}(m)=\frac{4}{a_{0}^{2}} \sin ^{2} \frac{\pi p}{N}+\frac{4}{a_{0}^{2}} \sinh ^{2} \frac{\pi m a}{N},
$$

where the integer $p$ runs over $0 \leq p \leq N-1$.

Defining $\theta_{p} \equiv \frac{2 \pi p}{N}$, the free energy on $C_{N}$ can be expressed as

$$
\begin{aligned}
F_{C_{N}} & =-\frac{1}{2} \int_{0}^{\infty} \frac{d t}{t} \sum_{p=0}^{N-1} e^{-a_{0}^{2} \bar{\lambda}_{p} t}=-\frac{1}{2} \int_{0}^{\infty} \frac{d t}{t} \sum_{p=0}^{N-1} e^{-\left(2-2 \cos \theta_{p}+4 \sinh ^{2} \frac{\pi m a}{N}\right) t} \\
& =-\frac{1}{2} \int_{0}^{\infty} \frac{d t}{t} \sum_{p=0}^{N-1} \sum_{l \in \mathbb{Z}} \cos l \theta_{p} I_{l}(2 t) e^{-2\left(\cosh \frac{2 \pi m a}{N}\right) t} \\
& =-\frac{1}{2} \int_{0}^{\infty} \frac{d t}{t} \sum_{l \in \mathbb{Z}} \sum_{p=0}^{N-1} e^{i l \theta_{p}} I_{l}(2 t) e^{-2\left(\cosh \frac{2 \pi m a}{N}\right) t} \\
& =-\frac{N}{2} \int_{0}^{\infty} \frac{d t}{t} \sum_{q \in \mathbb{Z}} I_{N q}(2 t) e^{-2\left(\cosh \frac{2 \pi m a}{N}\right) t} \\
& =-N \sum_{q=1}^{\infty} \int_{0}^{\infty} \frac{d t}{t} I_{N q}(2 t) e^{-2\left(\cosh \frac{2 \pi m a}{N}\right) t}-\frac{N}{2} \int_{0}^{\infty} \frac{d t}{t} I_{0}(2 t) e^{-2\left(\cosh \frac{2 \pi m a}{N}\right) t},
\end{aligned}
$$

where we have used the formula including the modified Bessel function of the first kind $I_{\nu}(z)[27]^{4}$

$$
e^{z \cos \theta}=\sum_{l \in \mathbb{Z}} \cos l \theta I_{l}(z),
$$

and $I_{l}(z)=I_{-l}(z)$ for $l \in \mathbb{Z}$.

By using the integration formula [27]

$$
\int_{0}^{\infty} \frac{d t}{t} I_{\nu}(2 t) e^{-2(\cosh x) t}=\frac{e^{-\nu x}}{\nu},
$$

\footnotetext{
${ }^{4}$ This expansion formula has been utilized for calculating vacuum energies in models of dimensional deconstruction $[28,29,30,31,32]$.
} 
we can evaluate the convergent sum in the first term in the last line of (24) as

$$
-N \sum_{q=1}^{\infty} \int_{0}^{\infty} \frac{d t}{t} I_{N q}(2 t) e^{-2\left(\cosh \frac{2 \pi m a}{N}\right) t}=-\sum_{q=1}^{\infty} \frac{\left(e^{-2 \pi m a}\right)^{q}}{q}=\ln \left(1-e^{-2 \pi m a}\right) .
$$

Since the second term in the last line of (24) diverges, we should regularize this contribution. We interpret this presentation as

$$
-\frac{N}{2} \lim _{\epsilon \rightarrow 0} \int_{0}^{\infty} \frac{d t}{t} I_{\epsilon}(2 t) e^{-2\left(\cosh \frac{2 \pi m a}{N}\right) t}
$$

Then, this reads

$$
-\frac{N}{2} \lim _{\epsilon \rightarrow 0} \frac{e^{-\frac{2 \pi m a}{N} \epsilon}}{\epsilon}=-\frac{N}{2} \lim _{\epsilon \rightarrow 0} \frac{1}{\epsilon}\left(1-\frac{2 \pi m a}{N} \epsilon\right)=-\frac{N}{2} \lim _{\epsilon \rightarrow 0} \frac{1}{\epsilon}+\pi m a .
$$

Dropping the first divergent term in (29), we can obtain the regularized free energy on $C_{N}$

$$
F_{C_{N}}=\ln \left(1-e^{-2 \pi m a}\right)+\pi m a=\ln (2 \sinh \pi m a) .
$$

Now, this expression is the same as that of free energy on $S^{1}$. Our choice (22) of $\mu$ implies the $N$-independent free energy.

\section{Heat kernel traces on odd spheres and their trigonometric analogue}

In this section, first we compute the trace of the heat kernel of scalar field theories on odd dimensional spheres. Next we propose the trigonometric analogue of the heat kernel traces.

\section{1 eigenvalues and degeneracies on odd spheres}

For $d \geq 2$, let $S^{d}$ be the $d$-dimensional sphere with the standard metric $g_{\mu \nu}(\mu, \nu=$ $1, \ldots, d)$, and let $\Delta$ be the Laplace-Beltrami operator on the space of smooth functions.

Consider the action for a scalar field in the continuum on $S^{d}$ given by

$$
S=\frac{1}{2} \int d^{d} x \sqrt{g}\left[\nabla_{\mu} \phi \nabla^{\mu} \phi+\left(m^{2}+\xi \mathcal{R}\right) \phi^{2}\right],
$$

where $g=\operatorname{det} g_{\mu \nu}$. The Laplacian acting on the scalar field is

$$
\Delta=\nabla^{\mu} \nabla_{\mu}=\frac{1}{\sqrt{g}} \partial_{\mu} \sqrt{g} g^{\mu \nu} \partial_{\nu}
$$

The scalar curvature $\mathcal{R}$ takes the value

$$
\mathcal{R}=\frac{d(d-1)}{a^{2}},
$$

on $S^{d}$ with the radius $a$. The Laplacian has a discrete and infinite spectrum. The eigenvalue of the operator $-\Delta+m^{2}+\xi \mathcal{R}$ is known to be [33]

$$
\begin{aligned}
a^{2} \lambda_{l} & =l(l+d-1)+\xi d(d-1)+m^{2} a^{2} \\
& =\left(l+\frac{d-1}{2}\right)^{2}-\frac{(d-1)^{2}}{4}+\xi d(d-1)+m^{2} a^{2},
\end{aligned}
$$


where $l$ is a non-negative integer, i.e., $l=0,1,2, \ldots$.

We consider two specific cases of the coupling $\xi$ to the scalar curvature $\mathcal{R}$. One is the case with the conformal coupling

$$
\xi=\xi_{d}=\frac{d-2}{4(d-1)}
$$

In this case, the eigenvalue becomes

$$
\begin{aligned}
a^{2} \lambda_{l} & =\left(l+\frac{d-2}{2}\right)\left(l+\frac{d}{2}\right)+m^{2} a^{2} \\
& =\left(l+\frac{d-1}{2}\right)^{2}-\frac{1}{4}+m^{2} a^{2} .
\end{aligned}
$$

It is known that the scalar field theory with the coupling $\xi_{d}$ has conformal invariance at zero mass $(m=0)$.

Another case is with the 'pseudo-conformal coupling' defined as

$$
\xi=\xi_{d+1}=\frac{d-1}{4 d} .
$$

In this case, the eigenvalue can be written as

$$
a^{2} \lambda_{l}=\left(l+\frac{d-1}{2}\right)^{2}+m^{2} a^{2} .
$$

The corresponding degeneracy (multiplicity) of the $l$ th eigenvalue is independent of $\xi$ and given by [33]

$$
g_{l}=\frac{2\left(l+\frac{d-1}{2}\right)}{(d-1) !} \prod_{n=1}^{d-2}(l+n) .
$$

Especially, for any $d$-sphere, we find

$$
g_{0}=1
$$

For instance, the expression (39) reads

$$
g_{l}=(l+1)^{2}, \quad \text { for } d=3,
$$

and

$$
g_{l}=\frac{1}{12}(l+2)^{2}(l+3)(l+1)=\frac{1}{12}(l+2)^{2}\left[(l+2)^{2}-1\right], \quad \text { for } d=5 .
$$

The degeneracy for odd spheres can be rewritten as [34]

$$
g_{l}=\frac{2(l+v)^{2}}{(2 v) !} \prod_{n=1}^{v-1}\left[(l+v)^{2}-n^{2}\right],
$$

where we set

$$
v \equiv \frac{d-1}{2} .
$$

Note that $v$ is a positive integer for odd $d$. 
Then, the heat kernel trace of massless conformal scalar field on $S^{d}$ ( $d$ odd) can be expressed as

$$
\begin{aligned}
\kappa_{v}(t) & =\sum_{l=0}^{\infty} g_{l} \exp \left\{-a^{2} \lambda_{l} t\right\} \\
& =\sum_{l=0}^{\infty} \frac{2(l+v)^{2}}{(2 v) !} \prod_{n=1}^{v-1}\left[(l+v)^{2}-n^{2}\right] \exp \left\{-\left[(l+v)^{2}-\frac{1}{4}\right] t\right\} \\
& =\sum_{l=v}^{\infty} \frac{2 l^{2}}{(2 v) !} \prod_{n=1}^{v-1}\left(l^{2}-n^{2}\right) \exp \left[-\left(l^{2}-\frac{1}{4}\right) t\right] \\
& =\frac{1}{2} \sum_{l \in \mathbb{Z}} \frac{2 l^{2}}{(2 v) !} \prod_{n=1}^{v-1}\left(l^{2}-n^{2}\right) \exp \left[-\left(l^{2}-\frac{1}{4}\right) t\right] .
\end{aligned}
$$

The last equality is due to $l^{2} \prod_{n=1}^{v-1}\left(l^{2}-n^{2}\right)=0$ for $l=0,1, \ldots, v-1$.

In the next subsection, we consider a trigonometric analogue of the heat kernel traces on odd spheres.

\section{2 trigonometric deformation}

We first consider a trigonometric analogue of eigenvalues of operators. Following the naive correspondence between the cases with $S^{1}$ and $C_{N}$, we replace eigenvalues for each case as follows.

For the case with conformal coupling, we replace the eigenspectrum as

$$
\begin{aligned}
a_{0}^{2} \bar{\lambda}_{p} & =4 \sin \frac{\pi}{N}\left(p+\frac{d-2}{2}\right) \sin \frac{\pi}{N}\left(p+\frac{d}{2}\right)+\mu^{2} a_{0}^{2} \\
& =2\left[\cos \frac{\pi}{N}-\cos \frac{2 \pi}{N}\left(p+\frac{d-1}{2}\right)\right]+\mu^{2} a_{0}^{2},
\end{aligned}
$$

where $\mu$ and $a_{0}$ is constants corresponding to the mass of the scalar field and the radius of $S^{d}$, respectively. Here $N$ is an arbitrary integer, which is considered here to be sufficiently large. For the case with pseudo-conformal coupling, we set

$$
\begin{aligned}
a_{0}^{2} \bar{\lambda}_{p} & =4 \sin ^{2} \frac{\pi}{N}\left(p+\frac{d-1}{2}\right)+\mu^{2} a_{0}^{2} \\
& =2\left[1-\cos \frac{2 \pi}{N}\left(p+\frac{d-1}{2}\right)\right]+\mu^{2} a_{0}^{2} .
\end{aligned}
$$

In both two cases, $p$ runs from 0 to $N-1$. Incidentally, these replacement is equivalent to considering $q$-analogue of $x,[x]=\frac{\sin \frac{\pi x}{N}}{\sin \frac{\pi}{N}} \cdot 5$

We also propose that the degeneracy is also deformed. In order to normalize the degeneracy which satisfies $g_{0}=1$, we consider the following form:

$$
\bar{g}_{p} \equiv \frac{\check{g}_{p}}{\check{g}_{0}},
$$

where

$$
\check{g}_{p}=\sin \frac{\pi}{N}(p+v) \prod_{n=-(v-1)}^{v-1} \sin \frac{\pi}{N}(p+v+n)
$$

\footnotetext{
${ }^{5}$ We have not to care about the normalization due to $\sin \frac{\pi}{N}$ etc., because they can be absorbed into the redefinition of the parameter $t$.
} 


$$
\begin{aligned}
& =\frac{1}{2^{v}}\left[1-\cos \frac{2 \pi}{N}(p+v)\right] \prod_{n=1}^{v-1}\left[\cos \frac{2 \pi n}{N}-\cos \frac{2 \pi}{N}(p+v)\right] \\
& =\frac{1}{2^{v}} \prod_{n=0}^{v-1}\left[\cos \frac{2 \pi n}{N}-\cos \frac{2 \pi}{N}(p+v)\right] .
\end{aligned}
$$

Note that $g_{p}>0$ for $p=0, \ldots, N-1$.

Now, the trigonometric deformation of the heat kernel trace of the massless conformal coupling scalar field on $S^{d}$ can be written as

$$
\begin{aligned}
\bar{\kappa}_{v}(t) & =\frac{1}{2} \sum_{p=0}^{N-1} \prod_{n=0}^{v-1} \frac{\cos \frac{2 \pi n}{N}-\cos \frac{2 \pi}{N}(p+v)}{\cos \frac{2 \pi n}{N}-\cos \frac{2 \pi v}{N}} \exp \left\{-2\left[\cos \frac{\pi}{N}-\cos \frac{2 \pi}{N}(p+v)\right] t\right\} \\
& =\frac{1}{2} \sum_{p=0}^{N-1} \prod_{n=0}^{v-1} \frac{\cos \theta_{n}-\cos \theta_{p}}{\cos \theta_{n}-\cos \theta_{v}} \exp \left[-2\left(\cos \frac{\pi}{N}-\cos \theta_{p}\right) t\right]
\end{aligned}
$$

where $\theta_{k} \equiv \frac{2 \pi}{N} k$. Similarly the case with pseudo-conformal coupling is treated. In the last equality in (50), permutation invariance of the trace (sum over $N$ terms) under $p \rightarrow p+$ (any integer) has been used. Note that the factor $1 / 2$ in front of the summation is needed because of $\cos \theta_{p}=\cos \theta_{N-p}$.

One can check that, in the limit of $N \rightarrow \infty$, the deformed heat kernel trace becomes the one on $S^{d}$, up to the redefinition of the parameter $t$.

\section{Free energies on odd spheres and their trigono- metric deformations}

In this section, we first explicitly demonstrate the calculation of the free energy on $S^{3}$ by using the heat kernel traces, of standard and deformed. Next, we give the regularized 'deformed' free energies of general odd dimensions.

\section{$5.1 \quad$ free energy on $S^{3}$}

First, we show the evaluation of the free energy on $S^{3}$ via the heat kernel trace method. Using the Poisson summation formula, we have

$$
\begin{aligned}
\sum_{l \in \mathbb{Z}} l^{2} e^{-l^{2} t} & =-\frac{\partial}{\partial t} \sum_{l \in \mathbb{Z}} e^{-l^{2} t}=-\frac{\partial}{\partial t}\left(\sqrt{\frac{\pi}{t}} \sum_{l \in \mathbb{Z}} e^{-\frac{\pi^{2} l^{2}}{t}}\right) \\
& =\frac{\sqrt{\pi}}{2 t^{3 / 2}} \sum_{l \in \mathbb{Z}} e^{-\frac{\pi^{2} l^{2}}{t}}-\frac{\pi^{5 / 2}}{t^{5 / 2}} \sum_{l \in \mathbb{Z}} l^{2} e^{-\frac{\pi^{2} l^{2}}{t}} .
\end{aligned}
$$

For the conformal coupling case, if we define $y^{2} \equiv \mu^{2} a^{2}-\frac{1}{4}$, we can proceed to calculate the free energy with the above relation, as

$$
\begin{aligned}
F_{3} & =-\frac{1}{2} \int_{0}^{\infty} \frac{d t}{t} \frac{1}{2} \sum_{l \in \mathbb{Z}} l^{2} e^{-l^{2} t-y^{2} t} \\
& =-\frac{\sqrt{\pi}}{8} \int_{0}^{\infty} \frac{d t}{t^{5 / 2}} \sum_{l \in \mathbb{Z}} e^{-y^{2} t-\frac{\pi^{2} l^{2}}{t}}+\frac{\pi^{5 / 2}}{4} \int_{0}^{\infty} \frac{d t}{t^{7 / 2}} \sum_{l \in \mathbb{Z}} l^{2} e^{-y^{2} t-\frac{\pi^{2} l^{2}}{t}} \\
& =-\frac{\sqrt{\pi}}{2} \sum_{l=0}^{\infty}\left(\frac{y}{\pi l}\right)^{3 / 2} K_{3 / 2}(2 \pi y l)-\frac{\sqrt{\pi}}{8} \int_{0}^{\infty} \frac{d t}{t^{5 / 2}} e^{-y^{2} t}
\end{aligned}
$$




$$
\begin{aligned}
& +\pi^{5 / 2} \sum_{l=0}^{\infty} l^{2}\left(\frac{y}{\pi l}\right)^{5 / 2} K_{5 / 2}(2 \pi y l) \\
= & -\frac{\sqrt{\pi}}{2} \sum_{l=0}^{\infty}\left(\frac{y}{\pi l}\right)^{3 / 2} \frac{1}{\sqrt{4 y l}}\left(1+\frac{1}{2 \pi y l}\right) e^{-2 \pi y l}-\frac{\sqrt{\pi}}{8} \Gamma\left(-\frac{3}{2}\right) y^{3} \\
& +\pi^{5 / 2} \sum_{l=0}^{\infty} l^{2}\left(\frac{y}{\pi l}\right)^{5 / 2} \frac{l^{2}}{\sqrt{4 y l}}\left(1+\frac{3}{2 \pi y l}+\frac{3}{4 \pi^{2} y^{2} l^{2}}\right) e^{-2 \pi y l} \\
= & \sum_{l=1}^{\infty}\left(\frac{y^{2}}{2 l}+\frac{y}{2 \pi l^{2}}+\frac{1}{4 \pi^{2} l^{3}}\right) e^{-2 \pi y l}-\frac{\pi}{6} y^{3} .
\end{aligned}
$$

Note that

$$
K_{3 / 2}(z)=\sqrt{\frac{\pi}{2 z}}\left(1+\frac{1}{z}\right) e^{-z}
$$

and

$$
K_{5 / 2}(z)=\sqrt{\frac{\pi}{2 z}}\left(1+\frac{3}{z}+\frac{3}{z^{2}}\right) e^{-z}
$$

For a massless conformal scalar field, we have to set $y \rightarrow \frac{i}{2}$. Then, we simply have

$$
\begin{aligned}
F_{3} & =\sum_{l=1}^{\infty}\left(-\frac{1}{8 l}+\frac{i}{4 \pi l^{2}}+\frac{1}{4 \pi^{2} l^{3}}\right)(-1)^{l}+\frac{i \pi}{48} \\
& =\left(\frac{\ln 2}{8}-\frac{i}{4 \pi} \eta(2)-\frac{1}{4 \pi^{2}} \eta(3)\right)+\frac{i \pi}{48} \\
& =\frac{\ln 2}{8}-\frac{i}{8 \pi} \zeta(2)-\frac{3}{16 \pi^{2}} \zeta(3)+\frac{i \pi}{48} \\
& =\frac{\ln 2}{8}-\frac{3 \zeta(3)}{16 \pi^{2}} .
\end{aligned}
$$

Note that

$$
\sum_{l=1}^{\infty} \frac{(-1)^{l-1}}{l}=\ln 2, \quad \sum_{l=1}^{\infty} \frac{(-1)^{l-1}}{l^{z}}=\frac{2^{z-1}-1}{2^{z-1}} \zeta(z) \equiv \eta(z) \quad(z \geq 2)
$$

where $\eta(z)$ is known as the Dirichlet eta function and $\zeta(z)$ is the Riemann zeta function. In particular, it is known that $\zeta(2)=\frac{\pi^{2}}{6}$.

The free energy of a massless pseudo-conformal scalar field on $S^{3}$ is obtained when we set $y=0$ in (52). Then, we find

$$
F_{3 p c}=\frac{\zeta(3)}{4 \pi^{2}} .
$$

Another derivation of free energies of massless pseudo-conformal scalar fields on odd sphere is exhibited in Appendix A.

\section{2 the deformation of free energies}

Next, we calculate the free energy by using the trigonometrically deformed heat kernel trace. We have already seen the expansion formula

$$
\sum_{p=0}^{N-1} e^{2 t \cos \theta_{p}}=N \sum_{q \in \mathbb{Z}} I_{N q}(2 t)
$$


where $\theta_{p}=\frac{2 \pi p}{N}$. By differentiating both side of the equation, we get

$$
\begin{aligned}
\sum_{p=0}^{N-1} \cos \theta_{p} e^{2 t \cos \theta_{p}} & =\frac{\partial}{\partial(2 t)} \sum_{p=0}^{N-1} e^{2 t \cos \theta_{p}}=N \sum_{q \in \mathbb{Z}} I_{N q}^{\prime}(2 t) \\
& =\frac{N}{2} \sum_{q \in \mathbb{Z}}\left[I_{N q-1}(2 t)+I_{N q+1}(2 t)\right]
\end{aligned}
$$

where we have used the recurrence relation

$$
I_{\nu}^{\prime}(z)=\frac{1}{2}\left[I_{\nu-1}(z)+I_{\nu+1}(z)\right],
$$

and $I_{\nu}^{\prime}(z)$ denotes the first derivative of $I_{\nu}(z)$. Then, we have the expression by an infinite series, which appears in the calculation of the free energy:

$$
\begin{aligned}
& \int_{0}^{\infty} \frac{d t}{t} \sum_{p=0}^{N-1}\left(1-\cos \theta_{p}\right) e^{2 t \cos \theta_{p}-2 t \cosh x} \\
= & N \int_{0}^{\infty} \frac{d t}{t} \sum_{q \in \mathbb{Z}}\left\{I_{N q}(2 t)-\frac{1}{2}\left[I_{N q-1}(2 t)+I_{N q+1}(2 t)\right]\right\} e^{-2 t \cosh x} .
\end{aligned}
$$

The integration of each term over $t$ can be performed by use of the formula (26). Thus we find

$$
\begin{aligned}
& \int_{0}^{\infty} \frac{d t}{t}\left\{I_{\nu}(2 t)-\frac{1}{2}\left[I_{\nu-1}(2 t)+I_{\nu+1}(2 t)\right]\right\} e^{-2 t \cosh x} \\
= & e^{-\nu x}\left[\frac{1}{\nu}-\frac{1}{2}\left(\frac{e^{x}}{\nu-1}+\frac{e^{-x}}{\nu+1}\right)\right] \\
= & e^{-\nu x}\left(\frac{1}{\nu}-\frac{\nu \cosh x}{\nu^{2}-1}-\frac{\sinh x}{\nu^{2}-1}\right)
\end{aligned}
$$

and then, we obtain

$$
\begin{aligned}
& \int_{0}^{\infty} \frac{d t}{t} \sum_{p=0}^{N-1}\left(1-\cos \theta_{p}\right) e^{2 t \cos \theta_{p}-2 t \cosh x} \\
= & 2 N \sum_{q=1}^{\infty} e^{-N q x}\left(\frac{1}{N q}-\frac{N q \cosh x}{N^{2} q^{2}-1}-\frac{\sinh x}{N^{2} q^{2}-1}\right) \\
& +N \lim _{\epsilon \rightarrow 0} e^{-\epsilon x}\left(\frac{1}{\epsilon}-\frac{\epsilon \cosh x}{\epsilon^{2}-1}-\frac{\sinh x}{\epsilon^{2}-1}\right) \\
= & 2 \sum_{q=1}^{\infty} e^{-N q x}\left(\frac{1}{q}-\frac{q \cosh x}{q^{2}-1 / N^{2}}-\frac{1}{N} \frac{\sinh x}{q^{2}-1 / N^{2}}\right) \\
& -N x+N \sinh x+N \lim _{\epsilon \rightarrow 0} \frac{1}{\epsilon},
\end{aligned}
$$

where we have replaced only the term of $q=0$ by the expression with $q=\epsilon \rightarrow 0$.

If we set $x \rightarrow \frac{i \pi}{N}$ in the above result,

$$
\begin{aligned}
& \int_{0}^{\infty} \frac{d t}{t} \sum_{p=0}^{N-1}\left(1-\cos \theta_{p}\right) e^{2 t \cos \theta_{p}-2 t \cos \frac{\pi}{N}} \\
= & 2 \sum_{q=1}^{\infty}(-1)^{q}\left(\frac{1}{q}-\frac{q \cos \frac{\pi}{N}}{q^{2}-1 / N^{2}}-\frac{i}{N} \frac{\sin \frac{\pi}{N}}{q^{2}-1 / N^{2}}\right) \\
& -i \pi+i N \sin \frac{\pi}{N}+N \lim _{\epsilon \rightarrow 0} \frac{1}{\epsilon}
\end{aligned}
$$


Using the summation formula [35],

$$
2 a^{2} \sum_{q=1}^{\infty} \frac{(-1)^{q}}{q^{2}-a^{2}}=1-\frac{\pi a}{\sin \pi a},
$$

we see the finite imaginary terms in (64) are canceled. The last term in the right-hand side of (64) is divergent but has no physical significance. We consider that the term should be dropped as a consequence of regularization. ${ }^{6}$

Now, the regularized free energy derived from the deformed heat kernel trace of the conformal scalar field on $S^{3}$ is given as ${ }^{7}$

$$
\begin{aligned}
\bar{F}_{3} & =\frac{1}{2\left(1-\cos \frac{2 \pi}{N}\right)} \sum_{q=1}^{\infty}(-1)^{q-1}\left(\frac{1}{q}-\frac{q \cos \frac{\pi}{N}}{q^{2}-1 / N^{2}}\right) \\
& =\frac{1}{2\left(1-\cos \frac{2 \pi}{N}\right)}\left[\ln 2-\cos \frac{\pi}{N}\left(\ln 2+\sum_{k=1}^{\infty} \frac{\eta(2 k+1)}{N^{2 k}}\right)\right] \\
& =\frac{\sin ^{2} \frac{\pi}{2 N}}{2 \sin ^{2} \frac{\pi}{N}} \ln 2-\frac{\cos \frac{\pi}{N}}{4 \sin ^{2} \frac{\pi}{N}} \sum_{k=1}^{\infty} \frac{\eta(2 k+1)}{N^{2 k}} \\
& =\frac{1}{8 \cos ^{2} \frac{\pi}{2 N}} \ln 2-\frac{\cos \frac{\pi}{N}}{4 \sin ^{2} \frac{\pi}{N}} \sum_{k=1}^{\infty} \frac{\eta(2 k+1)}{N^{2 k}} \\
& =\frac{\ln 2}{8}-\frac{\eta(3)}{4 \pi^{2}}+\frac{\pi^{2}}{N^{2}}\left(\frac{\ln 2}{32}+\frac{\eta(3)}{24 \pi^{2}}-\frac{\eta(5)}{4 \pi^{2}}\right)+O\left(\frac{\pi^{4}}{N^{4}}\right) \\
& \approx 0.0638+0.0230 \frac{\pi^{2}}{N^{2}}+O\left(\frac{\pi^{4}}{N^{4}}\right) .
\end{aligned}
$$

The free energy from the deformed heat kernel trace of the pseudo-conformal scalar field on $S^{3}$ is obtained by setting $x=0$ in the formulas used above. We find

$$
\begin{aligned}
\bar{F}_{3 p c} & =\frac{1}{2\left(1-\cos \frac{2 \pi}{N}\right)} \sum_{q=1}^{\infty}\left(\frac{q}{q^{2}-1 / N^{2}}-\frac{1}{q}\right) \\
& =\frac{1}{2 N^{2}\left(1-\cos \frac{2 \pi}{N}\right)} \sum_{q=1}^{\infty} \frac{1}{q\left(q^{2}-1 / N^{2}\right)} \\
& =\frac{1}{4 \sin ^{2} \frac{\pi}{N}} \sum_{k=1}^{\infty} \frac{\zeta(2 k+1)}{N^{2 k}} \\
& =\frac{\zeta(3)}{4 \pi^{2}}+\frac{\pi^{2}}{N^{2}}\left(\frac{\zeta(3)}{12 \pi^{2}}+\frac{\zeta(5)}{4 \pi^{4}}\right)+O\left(\frac{\pi^{4}}{N^{4}}\right) \\
& \approx 0.0304+0.0128 \frac{\pi^{2}}{N^{2}}+O\left(\frac{\pi^{4}}{N^{4}}\right) .
\end{aligned}
$$

The $N$-dependence of $\bar{F}_{3}$ and $\bar{F}_{3 p c}$ is plotted in FIG. 2. The limit of $N \rightarrow \infty$ yields $F_{3}$ and $F_{3 p c}$, respectively. The difference from the limiting value is one percent if $N \approx 20$ in each case.

\footnotetext{
${ }^{6}$ Namely, the regularization is consequently dropping the contribution from $I_{0}$ in the series and taking only the real part of the free energy.

${ }^{7}$ Another expression is $\bar{F}_{3}=\frac{1}{2\left(1-\cos \frac{2 \pi}{N}\right)}\left\{\ln 2+\frac{1}{2} \cos \frac{\pi}{N}[\beta(1 / N)+\beta(-1 / N)]\right\}, \beta(z) \equiv \frac{1}{2}[\psi((z+1) / 2)-$ $\psi(z / 2)]$, where $\psi(z)$ is the digamma function [27].
} 


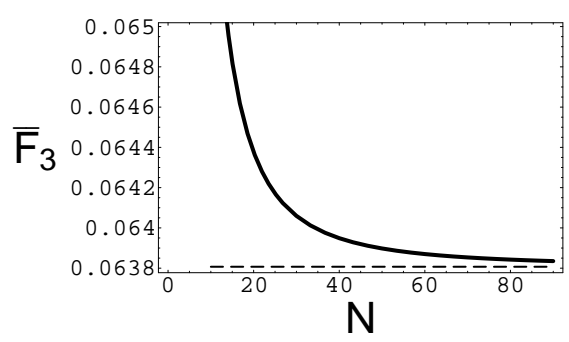

(a)

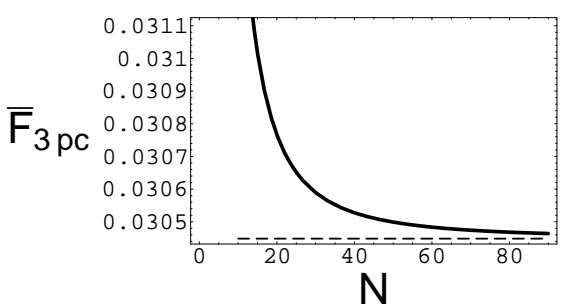

(b)

Figure 2: (a) $\bar{F}_{3}$ is plotted against $N$. The horizontal dashed line indicates $F_{3}$. (b) $\bar{F}_{3 p c}$ is plotted against $N$. The horizontal dashed line indicates $F_{3 p c}$.

For brevity, we hereafter call the free energy from the deformed heat kernel trace the 'deformed free energy'. In a similar manner, the deformed free energy of a conformal scalar field $\bar{F}_{5}$ can be calculated. Using the summation formula, we find

$$
\begin{aligned}
\int_{0}^{\infty} \frac{d t}{t} \sum_{p=0}^{N-1}\left(1-\cos \theta_{p}\right)\left(\cos \theta_{1}-\cos \theta_{p}\right) e^{2 t \cos \theta_{p}-2 t \cosh x} \\
=N \int_{0}^{\infty} \frac{d t}{t} \sum_{q \in \mathbb{Z}}\left\{-\frac{1}{2}\left(1+\cos \frac{2 \pi}{N}\right)\left[I_{N q-1}(2 t)-2 I_{N q}(2 t)+I_{N q+1}(2 t)\right]\right. \\
\left.+\frac{1}{4}\left[I_{N q-2}(2 t)-2 I_{N q}(2 t)+I_{N q+2}(2 t)\right]\right\} e^{-2 t \cosh x},
\end{aligned}
$$

and we finally obtain

$$
\begin{aligned}
\bar{F}_{5}= & -\frac{1}{4 \sin \frac{\pi}{N} \sin ^{2} \frac{2 \pi}{N} \sin \frac{3 \pi}{N}}\left[2 \sin ^{3} \frac{\pi}{2 N} \sin \frac{3 \pi}{2 N} \ln 2\right. \\
& \left.\quad+\sum_{k=1}^{\infty}\left\{\cos ^{3} \frac{\pi}{N}-2^{2(k-1)} \cos \frac{2 \pi}{N}\right\} \frac{\eta(2 k+1)}{N^{2 k}}\right] \\
= & -\frac{1}{2^{8}}\left(2 \ln 2+\frac{8 \eta(3)}{3 \pi^{2}}-\frac{16 \eta(5)}{\pi^{4}}\right) \\
& -\frac{1}{2^{8}} \frac{\pi^{2}}{N^{2}}\left(5 \ln 2+\frac{82 \eta(3)}{9 \pi^{2}}-\frac{40 \eta(5)}{3 \pi^{4}}-\frac{80 \eta(7)}{3 \pi^{6}}\right)+O\left(\frac{\pi^{4}}{N^{4}}\right) \\
\approx & -0.00574-0.0159 \frac{\pi^{2}}{N^{2}}+O\left(\frac{\pi^{4}}{N^{4}}\right) .
\end{aligned}
$$

In the limit of $N \rightarrow \infty$, one can find $\bar{F}_{5} \rightarrow F_{5}=-\frac{1}{2^{8}}\left(2 \ln 2+\frac{2 \zeta(3)}{\pi^{2}}-\frac{15 \zeta(5)}{\pi^{4}}\right)[36]$.

The deformed free energy of a pseudo-conformal scalar field $\bar{F}_{5 p c}$ is derived as

$$
\begin{aligned}
\bar{F}_{5 p c} & =-\frac{1}{4 \sin \frac{\pi}{N} \sin ^{2} \frac{2 \pi}{N} \sin \frac{3 \pi}{N}} \sum_{k=1}^{\infty}\left\{2^{2(k-1)}-\cos ^{2} \frac{\pi}{N}\right\} \frac{\zeta(2 k+1)}{N^{2 k}} \\
& =-\frac{\zeta(3)}{48 \pi^{2}}-\frac{\zeta(5)}{16 \pi^{4}}-\frac{\pi^{2}}{N^{2}}\left(\frac{\zeta(3)}{18 \pi^{2}}+\frac{5 \zeta(5)}{24 \pi^{4}}+\frac{5 \zeta(7)}{16 \pi^{6}}\right)+O\left(\frac{\pi^{4}}{N^{4}}\right) \\
& \approx-0.00320-0.00931 \frac{\pi^{2}}{N^{2}}+O\left(\frac{\pi^{4}}{N^{4}}\right) .
\end{aligned}
$$

In the limit of $N \rightarrow \infty$, one can find $\bar{F}_{5 p c} \rightarrow F_{5 p c}=-\frac{\zeta(3)}{48 \pi^{2}}-\frac{\zeta(5)}{16 \pi^{4}}$. 


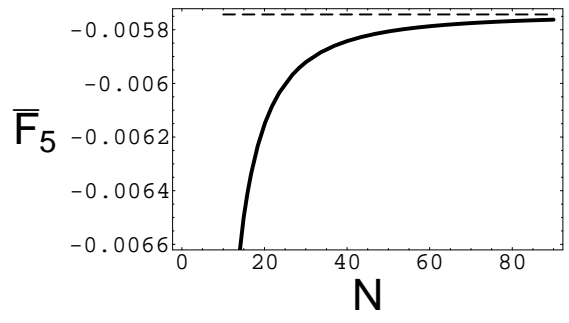

(a)

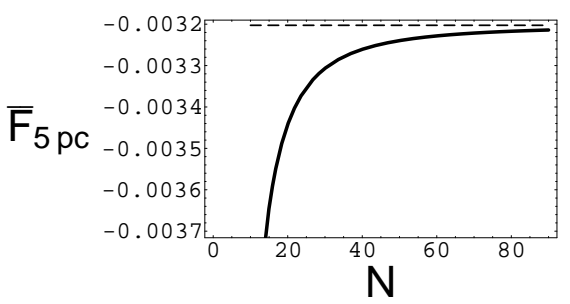

(b)

Figure 3: (a) $\bar{F}_{5}$ is plotted against $N$. The horizontal dashed line indicates $F_{5}$. (b) $\bar{F}_{5 p c}$ is plotted against $N$. The horizontal dashed line indicates $F_{5 p c}$.

The $N$-dependence of $\bar{F}_{5}$ and $\bar{F}_{5 p c}$ is plotted in FIG. 3. The limit of $N \rightarrow \infty$ yields $F_{5}$ and $F_{5 p c}$, respectively. The difference from the limiting value is one percent if $N \approx 40$ in each case.

For a general odd dimension $d$, we find that the regularized expressions of the deformed free energies take the form: ${ }^{8}$

$$
\begin{aligned}
\bar{F}_{d} & =-\frac{1}{V_{v}\left(\cos \theta_{v}\right)} \sum_{n=0}^{v} \frac{b_{v n}}{2^{n}} \sum_{r=0}^{\left\lfloor\frac{n-1}{2}\right\rfloor}\left(\begin{array}{c}
n \\
r
\end{array}\right) \sum_{q=1}(-1)^{q}\left(\frac{q \cos \frac{\pi(n-2 r)}{N}}{q^{2}-\frac{(n-2 r)^{2}}{N^{2}}}-\frac{1}{q}\right) \\
& =\frac{1}{2 V_{v}\left(\cos \theta_{v}\right)} \sum_{n=1}^{v} b_{v n} \operatorname{Re}\left[\int_{0}^{\infty} \frac{N}{e^{N \tau}+1} \cosh ^{n}\left(\tau-i \frac{\pi}{N}\right) d \tau\right] \\
& =\frac{1}{2 V_{v}\left(\cos \theta_{v}\right)} \int_{0}^{\infty} \frac{1}{e^{\tau}+1} \operatorname{Re}\left[V_{v}\left(\cosh \frac{\tau-i \pi}{N}\right)\right] d \tau
\end{aligned}
$$

and

$$
\begin{aligned}
\bar{F}_{d p c} & =-\frac{1}{N^{2} V_{v}\left(\cos \theta_{v}\right)} \sum_{n=1}^{v} \frac{b_{v n}}{2^{n}} \sum_{r=0}^{\left\lfloor\frac{n-1}{2}\right\rfloor}\left(\begin{array}{c}
n \\
r
\end{array}\right) \sum_{q=1} \frac{(n-2 r)^{2}}{q\left(q^{2}-\frac{(n-2 r)^{2}}{N^{2}}\right)} \\
& =\frac{1}{2 V_{v}\left(\cos \theta_{v}\right)} \sum_{n=1}^{v} n b_{v n} \int_{0}^{\infty} \ln \left(1-e^{-N \tau}\right) \cosh ^{n} \tau \tanh \tau d \tau \\
& =\frac{1}{2 V_{v}\left(\cos \theta_{v}\right)} \int_{0}^{\infty} \ln \left(1-e^{-N \tau}\right) V_{v}^{\prime}(\cosh \tau) \sinh \tau d \tau \\
& =-\frac{1}{2 V_{v}\left(\cos \theta_{v}\right)} \int_{0}^{\infty} \frac{N}{e^{N \tau}-1} V_{v}(\cosh \tau) d \tau \\
& =-\frac{1}{2 V_{v}\left(\cos \theta_{v}\right)} \int_{0}^{\infty} \frac{1}{e^{\tau}-1} V_{v}\left(\cosh \frac{\tau}{N}\right) d \tau
\end{aligned}
$$

where

$$
V_{v}(\chi)=\prod_{n=0}^{v-1}\left(\cos \frac{2 \pi n}{N}-\chi\right), \quad V_{v}^{\prime}(\chi)=\frac{d V_{v}(\chi)}{d \chi}
$$

and

$$
b_{v n}=\left.\frac{1}{n !} \frac{d^{n}}{d \chi^{n}} V_{v}(\chi)\right|_{\chi=0},
$$

\footnotetext{
${ }^{8}$ To obtain the integral forms, we utilize the identity $\nu^{-1}=\int_{0}^{\infty} e^{-\nu \tau} d \tau$. For numerical calculations, the convergence property of numerical integrations is rather worse than that of the expressions in series sums.
} 
i.e.,

$$
V_{v}(\chi)=\sum_{n=0}^{v} b_{v n} \chi^{n} .
$$

Note that $V_{v}(1)=\sum_{n=0}^{v} b_{n}=0$.

The large $N$ limit of the integral representation yields the normal free energy $F_{d}\left(F_{d p c}\right)$ on $S^{d}$ (with $d$ odd). This is shown in Appendix B.

\section{A comment about q-analogue}

The normal derivation of degeneracies of Laplacian eigenvalues on $S^{d}$ is based on the following expression in terms of the binomial coefficients:

$$
g_{l}=\left(\begin{array}{c}
d+l \\
d
\end{array}\right)-\left(\begin{array}{c}
d+l-2 \\
d
\end{array}\right)=\frac{(d+l) !}{d ! l !}-\frac{(d+l-2) !}{d !(l-2) !} .
$$

In the literature of $q$-analysis [20], the $q$-binomial coefficients are widely used. Thus, a natural deformation of the degeneracy is given by

$$
\bar{g}_{p}=\left[\begin{array}{c}
d+p \\
d
\end{array}\right]-\left[\begin{array}{c}
d+p-2 \\
d
\end{array}\right]=\frac{[d+p] !}{[d] ![p] !}-\frac{[d+p-2] !}{[d] ![p-2] !}
$$

where $[n] !=[n][n-1] \cdots[2][1]$. The use of $q=e^{i \frac{2 \pi}{N}}$ in this deformation yields

$$
\hat{g}_{p}=\frac{\cos \frac{\pi}{N}(l+v)}{\cos \frac{\pi}{N} v} \bar{g}_{p}
$$

The ambiguity in deformations comes from the fact that $[2 x] \neq[2][x] \neq 2[x]$.

Note that $\hat{g}_{p}$ can become negative. To obtain the heat kernel trace, we may restrict $0 \leq p \leq\lfloor N / 2\rfloor$, instead of dividing the trace by two. If we want to use permutation invariance to take a simple trace, we should consider the absolute value $\left|\hat{g}_{p}\right|$. Using the expansion

$$
\left|\cos \frac{\pi p}{N}\right|=\sqrt{1-\sin ^{2} \frac{\pi p}{N}}=1-\frac{1}{2} \sin ^{2} \frac{\pi p}{N}-\cdots,
$$

we can evaluate the $q$-deformed free energy using the degeneracy $\left|\hat{g}_{p}\right|$, up to $O\left(N^{-2}\right)$. For example,

$$
\begin{aligned}
\hat{F}_{3} & =\frac{\ln 2}{8}-\frac{\eta(3)}{4 \pi^{2}}+\frac{\pi^{2}}{N^{2}}\left(\frac{5 \ln 2}{64}-\frac{13 \eta(3)}{48 \pi^{2}}-\frac{5 \eta(5)}{8 \pi^{2}}\right)+O\left(\frac{\pi^{4}}{N^{4}}\right) \\
& \approx 0.0638+0.0232 \frac{\pi^{2}}{N^{2}}+O\left(\frac{\pi^{4}}{N^{4}}\right) .
\end{aligned}
$$

and

$$
\begin{aligned}
\hat{F}_{3 p c} & =\frac{\zeta(3)}{4 \pi^{2}}+\frac{\pi^{2}}{N^{2}}\left(\frac{5 \zeta(3)}{24 \pi^{2}}+\frac{5 \zeta(5)}{8 \pi^{2}}\right)+O\left(\frac{\pi^{4}}{N^{4}}\right) \\
& \approx 0.0304+0.0320 \frac{\pi^{2}}{N^{2}}+O\left(\frac{\pi^{4}}{N^{4}}\right) .
\end{aligned}
$$

The deviation at finite $N$ from the nondeformed free energy becomes even slightly larger than that in the case of our deformed free energy shown in the previous section. 


\section{Conclusion and prospect}

In the present paper, we first computed the free energy of a massive scalar field on a cycle graph $C_{N}$ by the heat kernel trace technique. Motivated by the result, we calculated the trigonometric deformed version of the free energy of a single conformally coupled free scalar field and that of a single pseudo-conformally coupled free scalar field on odd spheres. We showed that the values of the deformed free energies, obtained through the suitable regularization, approach the canonical values in the limit $N \rightarrow \infty$.

As an analogue calculation, the deformation of the heat kernel trace has many possible linkages to known theoretical models.

The calculations in the fuzzy space models [37, 38] and the quantum geometry models [39] have a common feature that the heat kernel trace consists of the finite number of eigenvalues in the models, though the degeneracy in such models is still integer. The models with $q$-deformed extra dimensions [40] and the statistical models with $q$-deformed algebra [41] may lead to similar forms of heat kernel traces.

Although we have not yet convinced of the precise relation to our calculation with noninteger degeneracies, we suppose that some models such as the theory with deformed phase spaces [42, 43], the nonlinear field space theory [44], field theories on fractals [45], and the unparticle models [46, 47, 48, 49, 50] might induce novel spectral densities and nonstandard heat kernel traces. The study on their appropriate regularization is very interesting. Seeking similarities to the heat kernels considered in random walks and quantum walks [51] is another subject of much interest.

It is interesting to imagine inverse problems on heat kernel traces. For example, we can ask whether there is an interacting scalar field theory on $S^{d}$ having the similar heat kernel trace, which approaches free theory if a certain number $N$ becomes large. We wish to discover the physical significance of the mathematical extension in such a sense.

The generalization of the deformation to other functions and manipulations is considered to be interesting. There are some $q$-analogues of the exponential function

$$
\sum_{n=0}^{\infty} \frac{x^{n}}{[n] !}, \quad \sum_{n=0}^{\infty} \frac{q^{\frac{n(n+1)}{2}} x^{n}}{[n] !}
$$

and the function used in the nonextensive statistical mechanics [52], in addition. Moreover, the standard integration over the parameter $t$ is even able to be changed along with the known extension in $q$-calculus [20]. These general treatments, however, would go beyond the scope of the present work.

\section{Appendix A}

In this Appendix A we present a direct calculation of the free energy of a pseudo-conformal scalar field on odd spheres.

First, we regularize the free energy by discarding $l=0$ in the summation in (45). For example of $d=3$, we take

$$
F_{3 p c}=-\frac{1}{2} \lim _{s \rightarrow 0} \sum_{l=1}^{\infty} l^{2} \int_{0}^{\infty} \frac{d t}{t^{1-s}} e^{-l^{2} t} .
$$

Next, we interpret the integration over $t$ here as ' $\Gamma(0)$ ', and proceed as follows:

$$
F_{3 p c}=-\frac{1}{2} \lim _{s \rightarrow 0} \Gamma(s) \sum_{l=1}^{\infty} l^{2-2 s}=-\frac{1}{2} \lim _{s \rightarrow 0} \Gamma(s) \zeta(-2+2 s)
$$




$$
\begin{aligned}
& =-\frac{1}{2} \lim _{s \rightarrow 0}(-1+s) \Gamma(-1+s) \zeta(-2+2 s) \\
& =\frac{1}{2} \pi^{-5 / 2} \lim _{s \rightarrow 0} \Gamma\left(\frac{3}{2}-s\right) \zeta(3-2 s)=\frac{\zeta(3)}{4 \pi^{2}},
\end{aligned}
$$

where we have used the formula [21]

$$
\Gamma\left(\frac{z}{2}\right) \zeta(z)=\pi^{z-\frac{1}{2}} \Gamma\left(\frac{1-z}{2}\right) \zeta(1-z) .
$$

Now, we verify the previous result.

Similarly, a slight calculation shows the value of $F_{5 p c}$ as follows:

$$
\begin{aligned}
& F_{5 p c}=-\frac{1}{2} \lim _{s \rightarrow 0} \Gamma(s) \sum_{l=1}^{\infty} \frac{l^{2-2 s}\left(l^{2}-1\right)}{12}=-\frac{1}{24} \lim _{s \rightarrow 0} \Gamma(s)[\zeta(-4+2 s)-\zeta(-2+2 s)] \\
& =-\frac{1}{24} \lim _{s \rightarrow 0}[(-1+s)(-2+s) \Gamma(-2+s) \zeta(-4+2 s)-(-1+s) \Gamma(-1+s) \zeta(-2+2 s)] \\
& =-\frac{1}{24}\left[\frac{2}{\pi^{9 / 2}} \Gamma\left(\frac{5}{2}\right) \zeta(5)+\frac{1}{\pi^{5 / 2}} \Gamma\left(\frac{3}{2}\right) \zeta(3)\right]=-\frac{\zeta(3)}{48 \pi^{2}}-\frac{\zeta(5)}{16 \pi^{4}} .
\end{aligned}
$$

The results of calculations of $F_{d p c}$ with those of $F_{d}$ obtained by Klebanov, Pufu and Safdi [36] are shown in Table 1.

\begin{tabular}{l|l}
$d$ & $F_{d}($ Ref. [36] $)$ \\
\hline 3 & $\frac{1}{2^{4}}\left(2 \ln 2-\frac{3 \zeta(3)}{\pi^{2}}\right) \approx 0.0638$ \\
5 & $\frac{-1}{2^{8}}\left(2 \ln 2+\frac{2 \zeta(3)}{\pi^{2}}-\frac{15 \zeta(5)}{\pi^{4}}\right) \approx-5.74 \times 10^{-3}$ \\
7 & $\frac{1}{2^{12}}\left(4 \ln 2+\frac{82 \zeta(3)}{15 \pi^{2}}-\frac{10 \zeta(5)}{\pi^{4}}-\frac{63 \zeta(7)}{\pi^{6}}\right) \approx 7.97 \times 10^{-4}$ \\
9 & $\frac{-1}{2^{16}}\left(10 \ln 2+\frac{1588 \zeta(3)}{105 \pi^{2}}-\frac{2 \zeta(5)}{\pi^{4}}-\frac{126 \zeta(7)}{\pi^{6}}-\frac{255 \zeta(9)}{\pi^{8}}\right) \approx-1.31 \times 10^{-4}$ \\
11 & $\frac{1}{2^{20}}\left(28 \ln 2+\frac{7794 \zeta(3)}{175 \pi^{2}}+\frac{1940 \zeta(5)}{63 \pi^{4}}-\frac{1218 \zeta(7)}{5 \pi^{6}}-\frac{850 \zeta(9)}{\pi^{8}}-\frac{1023 \zeta(11)}{\pi^{10}}\right) \approx 2.37 \times 10^{-5}$ \\
& \\
$d$ & $F_{d p c}$ \\
\hline 3 & $\frac{\zeta(3)}{4 \pi^{2}} \approx 0.0304$ \\
5 & $-\frac{\zeta(3)}{48 \pi^{2}}-\frac{\zeta(5)}{16 \pi^{2}} \approx-3.20 \times 10^{-3}$ \\
7 & $\frac{\zeta(3)}{360 \pi^{2}}+\frac{\zeta(5)}{96 \pi^{4}}+\frac{\zeta(7)}{64 \pi^{6}} \approx 4.66 \times 10^{-4}$ \\
9 & $-\frac{\zeta(3)}{2240 \pi^{2}}-\frac{7 \zeta(5)}{3840 \pi^{4}}-\frac{\zeta(7)}{256 \pi^{6}}-\frac{\zeta(9)}{256 \pi^{8}} \approx-7.83 \times 10^{-5}$ \\
11 & $\frac{\zeta(3)}{12600 \pi^{2}}+\frac{41 \zeta(5)}{120960 \pi^{4}}+\frac{13 \zeta(7)}{15360 \pi^{6}}+\frac{\zeta(9)}{768 \pi^{8}}+\frac{\zeta(11)}{1024 \pi^{10}} \approx 1.43 \times 10^{-5}$
\end{tabular}

Table 1: The values of $F_{d}[36]$ and $F_{d p c}$.

The integral expression of $F_{d}$ has been known as [53]

$$
\tilde{F}_{d} \equiv-\sin \frac{\pi d}{2} F_{d}=\frac{1}{\Gamma(1+d)} \int_{0}^{1} d u u \sin \pi u \Gamma\left(\frac{d}{2}+u\right) \Gamma\left(\frac{d}{2}-u\right) .
$$

We happened to find the following similar expression of $F_{d p c}$ :

$$
\tilde{F}_{d p c} \equiv-\sin \frac{\pi d}{2} F_{d p c}=\frac{1}{\Gamma(1+d)} \int_{-1 / 2}^{1 / 2} d u u \sin \pi u \Gamma\left(\frac{d}{2}+u\right) \Gamma\left(\frac{d}{2}-u\right) .
$$

The derivation of these integral expressions is performed in Appendix B. 


\section{Appendix B}

In this Appendix B, we show that the integral representations in the last line of (71) and $(72)$ in Section 5 can be utilized to obtain the values of $F_{d}$ and $F_{d p c}$ respectively by taking the limit of $N \rightarrow \infty$.

To this end, we first observe

$$
V_{v}\left(\cos \theta_{v}\right) \rightarrow \frac{2^{v} \pi^{2 v}}{N^{2 v}} \prod_{n=0}^{v-1}\left(v^{2}-n^{2}\right)=\frac{2^{v-1} \pi^{2 v}}{N^{2 v}}(2 v) !
$$

and

$$
V_{v}\left(\cosh \frac{w}{N}\right) \rightarrow \frac{2^{v} \pi^{2 v}}{N^{2 v}} \prod_{n=0}^{v-1}\left[-n^{2}-\left(\frac{w}{2 \pi}\right)^{2}\right]=\frac{2^{v} \pi^{2 v}}{N^{2 v}} \frac{\Gamma\left(v-i \frac{w}{2 \pi}\right) \Gamma\left(v+i \frac{w}{2 \pi}\right)}{\Gamma\left(-i \frac{w}{2 \pi}\right) \Gamma\left(i \frac{w}{2 \pi}\right)},
$$

at large $N$. Then, we get

$$
\begin{aligned}
& \lim _{N \rightarrow \infty} \bar{F}_{d}=F_{d} \\
& =-\frac{(-1)^{v}}{(2 v) !} \operatorname{Re}\left[\int_{0}^{\infty} \Gamma(v-i(\tau-i / 2)) \Gamma(v+i(\tau-i / 2))(\tau-i / 2) e^{-\pi\left(\tau-\frac{i}{2}\right)} d \tau\right]
\end{aligned}
$$

and

$$
\lim _{N \rightarrow \infty} \bar{F}_{d p c}=F_{d p c}=-\frac{(-1)^{v}}{(2 v) !} \int_{0}^{\infty} \Gamma(v-i \tau) \Gamma(v+i \tau) t e^{-\pi \tau} d \tau .
$$

To obtain these expressions, we have employed the identities [21]

$$
\Gamma(1-z) \Gamma(z)=\frac{\pi}{\sin \pi z}, \quad \Gamma(z+1)=z \Gamma(z) .
$$

Their relations to the other expressions (87) and (88) in Appendix A can be found by considering integrals in complex domain.

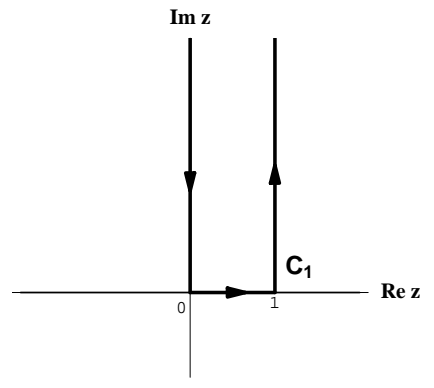

(a)

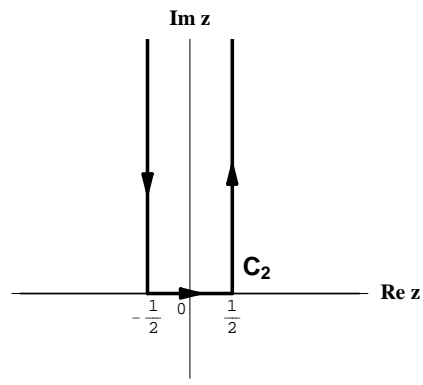

(b)

Figure 4: Contours $\mathrm{C}_{1}$ (a) and $\mathrm{C}_{2}(\mathrm{~b})$.

To find the relation on $F_{d}$, consider the integral

$$
\int_{\mathrm{C}_{1}} \Gamma\left(v+\frac{1}{2}-z\right) \Gamma\left(v+\frac{1}{2}+z\right) z e^{i \pi z} d z,
$$

with $\mathrm{C}_{1}$ the contour of FIG. 4(a). Because of the absence of poles inside the contour, the integral vanishes. The fact leads to

$$
\begin{aligned}
& -\int_{0}^{\infty} \Gamma\left(v+\frac{1}{2}-i \tau\right) \Gamma\left(v+\frac{1}{2}+i \tau\right)(i \tau) e^{-\pi \tau} i d \tau \\
& +\int_{0}^{1} \Gamma\left(v+\frac{1}{2}-u\right) \Gamma\left(v+\frac{1}{2}+u\right) u e^{i \pi u} d u \\
& +\int_{0}^{\infty} \Gamma\left(v+\frac{1}{2}-1-i \tau\right) \Gamma\left(v+\frac{1}{2}+1+i \tau\right)(i \tau+1)(-1) e^{-\pi \tau} i d \tau=0 .
\end{aligned}
$$


Arranging the equality, we obtain

$$
\begin{aligned}
& \int_{0}^{1} \Gamma\left(v+\frac{1}{2}-u\right) \Gamma\left(v+\frac{1}{2}+u\right) u e^{i \pi u} d u \\
& =-\int_{0}^{\infty} \tau\left(v-\frac{1}{2}-i \tau\right) \Gamma\left(v-\frac{1}{2}-i \tau\right) \Gamma\left(v+\frac{1}{2}+i \tau\right) e^{-\pi \tau} d \tau \\
& -\int_{0}^{\infty}(\tau-i)\left(v+\frac{1}{2}+i \tau\right) \Gamma\left(v-\frac{1}{2}-i \tau\right) \Gamma\left(v+\frac{1}{2}+i \tau\right) e^{-\pi t} d \tau \\
& =-(2 v+1) \int_{0}^{\infty} \Gamma\left(v-\frac{1}{2}-i \tau\right) \Gamma\left(v+\frac{1}{2}+i \tau\right)\left(\tau-\frac{i}{2}\right) e^{-\pi \tau} d \tau
\end{aligned}
$$

Comparing the imaginary part of each side of (96), we find that Eq. (87) is equivalent to Eq. (91).

To find the relation on $F_{d p c}$, consider the similar integral

$$
\int_{\mathrm{C}_{2}} \Gamma\left(v+\frac{1}{2}-z\right) \Gamma\left(v+\frac{1}{2}+z\right) z e^{i \pi z} d z=0
$$

with $\mathrm{C}_{2}$ the contour of FIG. 4(b). This leads to

$$
\begin{aligned}
& -\int_{0}^{\infty} \Gamma\left(v+\frac{1}{2}+\frac{1}{2}-i \tau\right) \Gamma\left(v+\frac{1}{2}-\frac{1}{2}+i \tau\right)\left(i \tau-\frac{1}{2}\right)(-i) e^{-\pi \tau} i d \tau \\
& +\int_{-1 / 2}^{1 / 2} \Gamma\left(v+\frac{1}{2}-u\right) \Gamma\left(v+\frac{1}{2}+u\right) u e^{i \pi u} d u \\
& +\int_{0}^{\infty} \Gamma\left(v+\frac{1}{2}-\frac{1}{2}-i \tau\right) \Gamma\left(v+\frac{1}{2}+\frac{1}{2}+i \tau\right)\left(i \tau+\frac{1}{2}\right)(i) e^{-\pi \tau} i d \tau=0
\end{aligned}
$$

Arranging the equality, we obtain

$$
\begin{aligned}
& \int_{-1 / 2}^{1 / 2} \Gamma\left(v+\frac{1}{2}-u\right) \Gamma\left(v+\frac{1}{2}+u\right) u e^{i \pi u} d u \\
& =\int_{0}^{\infty}\left(i \tau-\frac{1}{2}\right)(v-i \tau) \Gamma(v-i \tau) \Gamma(v+i \tau) e^{-\pi \tau} d \tau \\
& +\int_{0}^{\infty}\left(i \tau+\frac{1}{2}\right)(v+i \tau) \Gamma(v-i \tau) \Gamma(v+i \tau) e^{-\pi \tau} d \tau \\
& =i(2 v+1) \int_{0}^{\infty} \Gamma(v-i \tau) \Gamma(v+i \tau) \tau e^{-\pi \tau} d \tau .
\end{aligned}
$$

Comparing the imaginary part of each side of (99), we find that equations (88) and (92) are equivalent.

\section{References}

[1] G. V. Dunne, J. Phys. A41 (2008) 304006.

[2] D. V. Vassilevich, Phys. Rep. 388 (2003) 279.

[3] P. B. Gilkey, Invariance Theory, the Heat Equation, and the Atiyah-Singer Index Theorem (CRC Press, Florida, 1995).

[4] E. Elizalde, S. D. Odintsov, A. Romeo, A. A. Bytsenko and S. Zerbini, Zeta Regularization Techniques with Applications (World Scientific, Singapore, 1994). 
[5] E. Elizalde, Ten Physical Applications of Spectral Zeta Functions (Springer, Berlin, 1995).

[6] K. Kirsten, Spectral Functions in Mathematics and Physics (CRC Press, Florida, 2002).

[7] A. A. Bytsenko, G. Cognola, E. Elizalde, V. Moretti and S. Zerbini, Analytic Aspects of Quantum Fields (World Scientific, Singapore, 2003).

[8] J. S. Dowker and R. Critchley, Phys. Rev. D13 (1976) 3224.

[9] S. W. Hawking, Commun. Math. Phys. 55 (1977) 133.

[10] M. J. Duff and D. Toms, Kaluza-Klein-Kounterterms, in Unification of fundamental interactions II, S. Ferrara and J. Ellis eds. (Plenum, New York, 1982), p. 29.

[11] G. Calcagni, D. Oriti and J. Thürigen, Class. Quant. Grav. 30 (2013) 125006, and references therein.

[12] J. W. Barrett, S. Kerr and J. Louko, J. Phys. A46 (2013) 185201.

[13] S. Kerr, arXiv:1408.1449 [hep-th].

[14] S. Kerr, arXiv:1602.04264 [hep-th].

[15] R. C. Brower, G. Fleming, A. D. Gasbarro, T. Raben, C.-I. Tang and E. Weinberg, In Proceedings, 33rd International Symposium on Lattice Field Theory (Lattice 2015), arXiv:1601.01367 [hep-lat], and references therein.

[16] N. Kan and K. Shiraishi, J. Phys. A46 (2013) 365401.

[17] S. Matuura, T. Misumi and K. Ohta, Prog. Theor. Exp. Phys. 2014 (2014) 123 B01.

[18] S. Matuura, T. Misumi and K. Ohta, Prog. Theor. Exp. Phys. 2015 (2015) 033B07, and references therein.

[19] R. J. Wilson, Introduction to Graph Theory, 4-th ed. (Longman, New York, 1997).

[20] V. Kac and P. Cheung, Quantum Calculus (Springer, New York, 2002).

[21] I. S. Gradshtein and I. M. Ryzhik, Table of integrals, series and products (Academic Press, New York, 1980).

[22] A. Monin, Phys. Rev. D94 (2016) 085013.

[23] B. Mohar, The Laplacian spectrum of graphs, in Graph Theory, Combinatorics, and Applications, Y. Alavi et al. eds. (Wiley, New York, 1991), p. 871.

[24] B. Mohar, Discrete Math. 109 (1992) 171.

[25] B. Mohar, Some applications of Laplace eigenvalues of graphs, in Graph Symmetry, Algebraic Methods, and Applications, G. Hahn and G. Sabidussi eds. (Kluwer, Dordrecht, 1997), p. 225.

[26] R. Merris, Linear Algebra Appl. 197 (1994) 143.

[27] A. P. Prudnikov, Yu. A. Brychkov and O. I. Marichev, Integrals and Series, vol. 2: special functions (Gordon \& Breach Science Pub., New York, 1986).

[28] N. Kan, K. Sakamoto and K. Shiraishi, Eur. Phys. J. C28 (2003) 425.

[29] K. Shiraishi, K. Sakamoto and N. Kan, J. Phys. G29 (2003) 595.

[30] N. Kan and K. Shiraishi, Class. Quant. Grav. 20 (2003) 4965.

[31] N. Kan and K. Shiraishi, Prog. Theor. Phys. 111 (2004) 745.

[32] N. Kan and K. Shiraishi, Prog. Theor. Phys. 121 (2009) 1035. 
[33] A. Erdelyi, W. Magnus, F. Oberhettinger and F. G. Tricomi, Higher transcendental functions, vol. II (McGraw-Hill, New York, 1953).

[34] P. Candelas and S. Weinberg, Nucl. Phys. B237 (1984) 397.

[35] A. P. Prudnikov, Yu. A. Brychkov and O. I. Marichev, Integrals and Series, vol. 1: elementary functions (Gordon \& Breach Science Pub., New York, 1986).

[36] I. R. Klebanov, S. S. Pufu and B. R. Safdi, JHEP 1110 (2011) 038.

[37] N. Sasakura, JHEP 0412 (2004) 009.

[38] N. Sasakura, JHEP 0503 (2005) 015.

[39] G. Calcagni, D. Oriti and J. Thürigen, Class. Quant. Grav. 31 (2014) 135014.

[40] S. Naka and H. Toyoda Prog. Theor. Phys. 109 (2003) 103.

[41] A. Lavagno and P. Narayana Swamy, Physica A389 (2010) 993.

[42] T. P. Sotiriou, M. Visser and S. Weinfurtner, Phys. Rev. D84 (2011) 104018.

[43] M. Arzano, G. Gubitosi, J. Magueijo and G. Amelio-Camelia, Phys. Rev. D91 (2015) 125031.

[44] J. Mielczarek and T. Trześniewski, Phys. Lett. B759 (2016) 424.

[45] G. V. Dunne, J. Phys. A45 (2012) 374016.

[46] H. Georgi, Phys Rev. Lett. 98 (2007) 221601.

[47] M. A. Stephanov, Phys Rev. D76 (2007) 035008.

[48] J.-P. Lee, Phys Rev. D79 (2009) 076002.

[49] P. Nicolini and E. Spallucci, Phys. Lett. B695 (2011) 290.

[50] A. M. Frassino, P. Nicolini and O. Panella, arXiv:1311.7173 [hep-ph].

[51] J. Kempe, Contemporary Physics 44 (2003) 307.

[52] C. Tsallis, J. Stat. Phys. 52 (1988) 479.

[53] S. Giombi and I. R. Klebanov, JHEP 1503 (2015) 117. 